\title{
TOLERANCE BUDGETING IN A NOVEL COARSE-FINE STRATEGY FOR MICRO- ASSEMBLY
}

\author{
Vincent Henneken and Marcel Tichem \\ Precision Manufacturing and Assembly, 3mE, TU Delft, Mekelweg 2, $2628 \mathrm{CD}$ Delft, \\ The Netherlands
}

\begin{abstract}
This paper presents the tolerance budgeting for the coarse assembly step in a novel coarse-fine strategy for micro-assembly. The final assembly step is to be performed using MEMS-based functionality that remains part of the product. Total tolerance build-up due to the coarse assembly and dimensional inaccuracies has been determined for the alignment of a single mode optical fibre to a laser diode, a challenging problem in optical telecommunication.
\end{abstract}

Key words: tolerance budgeting; product-internal assembly functions; MEMS technology; fibre-chip coupling; silicon optical bench (SiOB); mechanical stop; fluid selfalignment

\section{INTRODUCTION}

The purpose of this paper is to discuss key issues concerning the coarse assembly step in a novel coarse-fine strategy for micro-assembly, of which the feasibility is being investigated in a structured approach. The need for reducing effort related to the handling of small parts explains the idea for focusing on quite different methods for assembly ${ }^{1}$. This research focuses on the investigation of the method of micro-assembly by means of productinternal assembly functions. In this method assembly functionality is created, which is integrated with the product to be assembled, and which remains as part of the product. This functionality includes part actuation, position sensing and part freezing. The method is applied in a two-stage process. In the first stage coarse positioning of components is achieved using productexternal assembly functions, typically by a (semi) automatic production machine or a human operator. The final, accurate positioning is performed 
on basis of product-internal assembly functions. Overall, this aims to result in lower total production cost and/or improved product quality. Micro Electro Mechanical System (MEMS) technology has been selected for use as supporting fabrication technology, because of the small attainable feature sizes and very high accuracy, and it is potentially low-cost due to its possibility of batch-wise processing. The potential use of MEMS-based structures for the purpose of micro-assembly has been reported on before (e.g. by $\mathrm{Syms}^{2}$ ), but its feasibility has never before been investigated in a structured approach. In this investigation it is the aim to research all aspects that influence under which circumstances product-internal functionality can be used for the assembly of that particular product. This paper mainly deals with issues related to the coarse assembly and with the interactions between the coarse and fine assembly functionality/steps, which play an important role within the method of micro-assembly using product-internal assembly functions, and which are closely connected with a high level of complexity. Describing this complexity and the possible choices with their accompanying consequences is the aim of this paper. Investigating these issues is considered very relevant for the attainability of success for the concept, and at the same time it can provide a broader insight in the assembly process in general.

\section{APPROACH}

The approach that is taken for the described exploratory research is that of a case investigation. The industrial case considered for investigation is the alignment of a single mode optical fibre to a laser diode, which is a challenging problem in optical telecommunication and sensing applications. This is embedded in the small dimensions of the laser output waveguide (typically $2 \mu \mathrm{m} \times 0.4 \mu \mathrm{m}$ ) and the optical fibre ( $8 \mu \mathrm{m}$ core diameter; overall fibre diameter; $125 \mu \mathrm{m}$ ). Alignment accuracy in the order of $0.1 \mu \mathrm{m}$ in the lateral dimension has to be maintained to achieve sufficient coupling efficiency over the economic lifetime of the system. Highly expensive machines or delicate manual handling are normally employed for the needed alignment and fixation steps, which last typically in the order of minutes. Normally, this is performed using active alignment, i.e. alignment while measuring the optical performance efficiency of the assembly.

In the project a number of concepts for thermal actuation of the fibre tip in one or two degrees of freedom perpendicular to the fibre optical axis have been developed. One of these developed concepts is used as starting point for the investigation of the coarse assembly process. This concept, which is depicted in Figure 1, concerns a horizontal planar layout in which the fibre is 
placed in an in-plane groove allowing two integrated thermal actuators to move the fibre tip in- and out-of-plane independently. The entire device is built up from one monolithic part, with silicon as base material. For its realisation highly accurate IC-compatible lithographical processing is used.

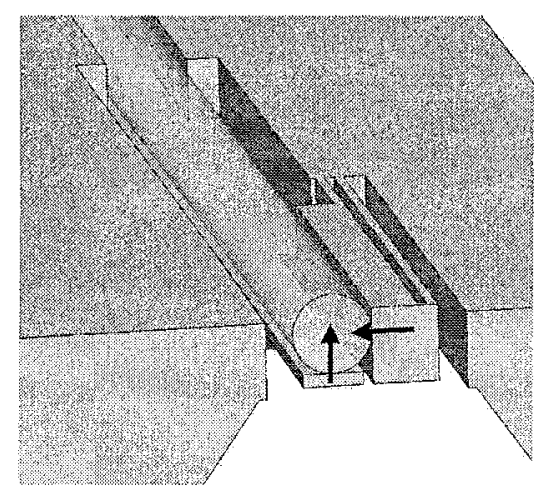

Figure 1. MEMS-based actuator concept for fibre tip positioning used as starting point in the coarse assembly investigation.

The first MEMS-based demonstrators for positioning the fibre in two degrees of freedom perpendicular to the fibre axis have readily been fabricated and are employed for investigating of (part of) the aspects listed here above. A more in-depth treatment of the design process has been covered in a previous publication ${ }^{3}$. A close-up SEM image of a fabricated fibre actuation structure is shown in Figure 2. The typical length of the actuators is around $1800 \mu \mathrm{m}$, with a total travel distance of $15-20 \mu \mathrm{m}$ in the directions perpendicular to the fibre axis. The final fine fixation is also under investigation within the project, but this aspect is not topic of the present paper.

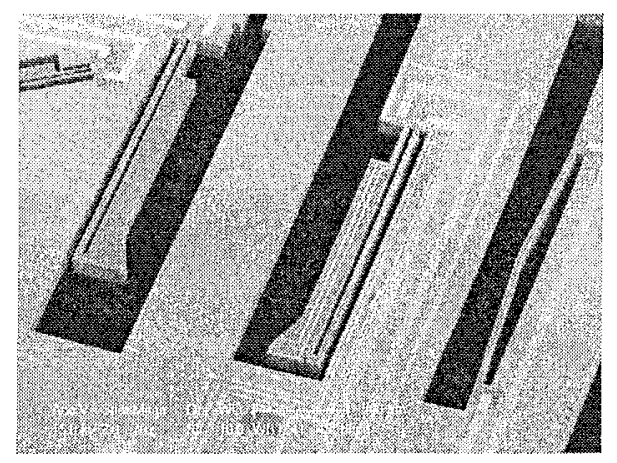

Figure 2. SEM image of fabricated in-plane fibre actuation structures. 
The tentative choices that have been made for the specific case under consideration will be presented in detail. Aim of the investigation is to develop micro-product and micro-assembly guidelines that have broader application than the specific optoelectronic case considered.

\section{TOLERANCE ANALYSIS FIBRE-CHIP COUPLING}

Tolerance build-up is the overall result of all decisions concerning the configuration, fabrication and assembly of the device. This section will successively deal with all three items for the specific case under consideration.

\subsection{Configuration}

In practice, positioning a part cannot be seen separately from its immediate environment. A position relation always must be established with respect to another part or to a base structure. In the case considered the important position relation is the optical path between the single mode fibre tip and the laser diode exit facet. For functional reasons it is not possible to connect both parts directly to each other. Instead this position relation needs to be established indirectly, thereby creating a part stack of which the tolerance build-up must be controlled such that the accuracy of the optical path remains within the desired range for each individual degree of freedom.

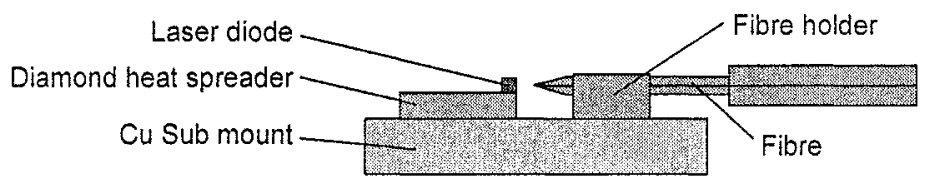

Figure 3. Conventional layout fibre coupled laser diode package.

A conventional layout is presented in Figure 3. A commonly used base structure (or sub mount) is copper due to its good heat conductivity. Optionally a diamond heat spreader is positioned in between the copper sub mount and the laser diode for even better heat regulation capabilities. It was found that the copper-diamond stack could be replaced with a single silicon layer without negatively influencing the thermal properties too much. Thereby opening up the possibility of creating lithography-based alignment features on the sub mount or potentially even integrating the sub mount and the actuator part into one single silicon part, which is from assembly point of 
view the most attractive alternative. However, integrating all assembly functionality into a single silicon component with the required accuracy using MEMS-based technology is very challenging. For this reason it was decided to focus on an assembly solution with separate silicon base part and actuator part (see Figure 4 for a schematic representation of this layout).

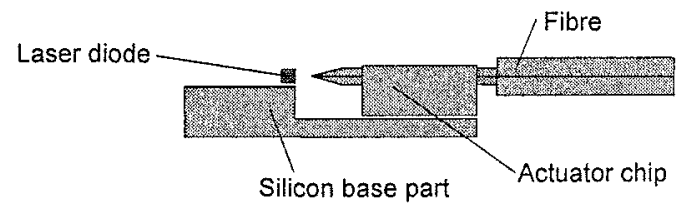

Figure 4. Proposed alternative layout fibre coupled laser diode package.

\subsection{Fabrication and assembly related issues}

Beside the configuration other tolerance build-up influencing components are fabrication and assembly related: dimensional inaccuracies, ranges of product-internal assembly process, parasitic tolerance build-up induced by internal actuator (only in the directions that are not fine positioned by the product-internal assembly functions), positioning inaccuracies, and shifts due to the fixation process. In the remaining part examples will be provided of each.

\subsubsection{Functional accuracy requirements}

As mentioned previously, the alignment tolerance between the laser diode and the fibre tip is very critical in the directions perpendicular to the fibre axis $( \pm 0.1 \mu \mathrm{m})$. According to the sign convention generally used in fibre to laser diode alignment these are the $\mathrm{x}$ and $\mathrm{y}$-directions, see Figure 5.

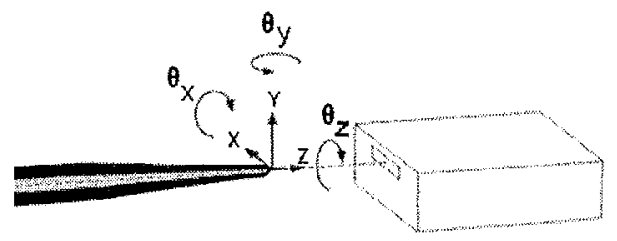

Figure 5. Sign convention fibre to laser alignment.

Naturally, the required accuracies are not the same in all directions. An overview of the required tolerances in all degrees of freedom is presented in 
the first column of Table 1. All values are displayed in upper (positive number) and lower (negative number) specification limits.

Table 1. Tolerance build-up for all individual degrees of freedom

\begin{tabular}{|c|c|c|c|c|c|}
\hline DOF & $\begin{array}{c}\text { Required } \\
\text { tolerance by } \\
\text { functionality } \\
\qquad \mathbf{i}_{\mathrm{req}}\end{array}$ & $\begin{array}{c}\text { Maximal } \\
\text { correction } \\
\text { by actuator } \\
\mathbf{i}_{\text {act }}\end{array}$ & $\begin{array}{l}\text { Parasitic } \\
\text { tolerance } \\
\text { build-up by } \\
\text { actuator } \\
\mathbf{i}_{\text {act, par }}\end{array}$ & $\begin{array}{c}\text { Cumulated } \\
\text { dimensional } \\
\text { tolerance } \\
\text { build-up } \\
\mathbf{\Sigma} \mathbf{i}_{\text {dim, inace }}\end{array}$ & $\begin{array}{c}\text { Maximal allowed } \\
\text { tolerance build-up } \\
\text { of coarse assembly } \\
\text { process } \\
\mathbf{\Sigma i}_{\text {CA, allow }}\end{array}$ \\
\hline $\mathbf{x}$ & $\pm 0.1 \mu \mathrm{m}$ & $\pm 10 \mu \mathrm{m}$ & - & $\pm 4.5 \mu \mathrm{m}$ & $\pm 5.5 \mu \mathrm{m}$ \\
\hline$y$ & $\pm 0.1 \mu \mathrm{m}$ & $\pm 10 \mu \mathrm{m}$ & - & $\pm 7.5 \mu \mathrm{m}$ & $\pm 2.5 \mu \mathrm{m}$ \\
\hline$z$ & $\pm 0.5 \mu \mathrm{m}$ & $\begin{array}{l}\text { Not aligned } \\
\text { by PIAF }\end{array}$ & Insignificant & Insignificant & $\pm 0.5 \mu \mathrm{m}$ \\
\hline$\theta_{x}$ & $\pm 1.5^{\circ}$ & $\begin{array}{l}\text { Not aligned } \\
\text { by PIAF }\end{array}$ & $-0.5^{\circ}-0^{\circ}$ & Insignificant & $-1^{\circ}+1.5^{\circ}$ \\
\hline$\theta_{y}$ & $\pm 1.5^{\circ}$ & $\begin{array}{l}\text { Not aligned } \\
\text { by PIAF }\end{array}$ & $-0.5^{\circ}-0^{\circ}$ & Insignificant & $-1^{\circ}+1.5^{\circ}$ \\
\hline \multirow[t]{2}{*}{$\theta_{\mathrm{z}}$} & $\begin{array}{l}\infty \\
\text { rotational } \\
\text { symmetric } \\
\text { fibre end } \\
\pm 10^{\circ}\end{array}$ & $\begin{array}{l}\text { Not aligned } \\
\text { by PIAF }\end{array}$ & - & Insignificant & $\infty$ \\
\hline & $\begin{array}{l}\text { non-rotational } \\
\text { symmetric } \\
\text { fibre-end }\end{array}$ & $\begin{array}{c}\text { Not aligned } \\
\text { by PIAF }\end{array}$ & - & Insignificant & $\pm 10^{\circ}$ \\
\hline
\end{tabular}

\subsubsection{Consequences product-internal assembly functionality}

As starting point for the coarse assembly investigation an actuator chip concept has been taken that is capable of positioning the fibre tip in the $\mathrm{x}$ and $y$-directions perpendicular to its optical axis. Although the required accuracy in z-direction is also quite strict, this limitation is made to control the complexity in fabrication of the MEMS-based fine assembly functionality to a manageable level.

The limited attainable complexity of (MEMS-based) fine assembly solutions has as a consequence that the coarse assembly functionality needs to take care of the final positioning in the remaining degrees of freedom. The attained positioning accuracy in these degrees of freedom should be at least equal to the required accuracy in these directions. For the other degrees of freedom this should at least be sufficient to enable successful final positioning by the product-internal micro actuation functionality.

The maximal correction by the product-internal actuators is $15-20 \mu \mathrm{m}$, but due to the fact that fine alignment of the fibre tip takes place by bending of the fibre end, unwanted parasitic tolerance build-up is induced in some of the unactuated directions. Of these, the rotations $\theta_{x}$ and $\theta_{y}$ of the fibre around 
the $\mathrm{x}$ and $\mathrm{y}$-axis are considered not negligible and are in the order of $0.5^{\circ}$ in the negative direction, see columns 2 and 3 in Table 1.

\subsubsection{Dimensional inaccuracies}

In order to be able to design the product-internal and the coarse assembly process, knowledge needs to be gathered on dimensional accuracies of all parts within the optical assembly stack. The optical assembly stack consists of a silicon sub mount, a GaAs distributed feedback (DFB) laser, a productinternal actuator chip and a single mode optical fibre. The intermediate bonds between these parts (where applicable) also account for part of the tolerance build-up, but these will be treated separately. The contributions of the individual parts to the total tolerance build-up due to dimensional accuracies are in a comparable range. For example, the tolerances on the outer dimensions of a typical laser diode are quite large, $\pm 10 \mu \mathrm{m}$, due to the fabrication processes used, but these are normally not employed in the assembly process. Instead, fiducial marks are used for the positioning of the laser diode in the horizontal plane, which are made using lithography with a typical accuracy of $\pm 1 \mu \mathrm{m}$ relative to the laser facet. When the laser diode is placed with the laser facet close to the bottom side ('junction-down'), then the position of the laser facet can vary only as much as $\pm 2 \mu \mathrm{m}$ in $\mathrm{y}$ direction.

For the actuator part and the silicon base part, the tolerances in $x$ and $y$ direction are also IC processing-based, and are therefore both in the same range as for the laser diode facet, $\pm 1 \mu \mathrm{m}$ and $\pm 2 \mu \mathrm{m}$ respectively.

Most tolerance build-up in the optical fibre is in the $\mathrm{x}$ and $\mathrm{y}$ direction due to core-cladding eccentricity. Maximal tolerance build-up between core and cladding is calculated to be $\pm 1.5 \mu \mathrm{m}$.

In the dimensional inaccuracy analysis the z-direction is not taken into account since the final accuracy in this direction will be achieved by positioning the fibre tip directly relative to the laser diode facet. Therefore any dimensional inaccuracies of the components are insignificant for reaching the accuracy in this direction. The cumulated tolerance build-up due to dimensional accuracies is displayed in column 4 of Table 1 . The rightmost column represents the maximum allowed tolerance build-up of the coarse assembly process. This can be read as a requirements list for the coarse assembly process; the coarse assembly process must comply with these limits in order to fulfil the overall assembly demands. It can be clearly seen that the demanded accuracy in the z-direction is now by far the most critical and is likely to deliver the largest challenge for the coarse assembly process. 


\subsubsection{Passive alignment structure fabrication}

Besides meeting the specific accuracy requirements cost is the main driving factor for the decision which positioning solutions will be employed for the individual degrees of freedom. The addition of product-internal assembly functionality is expected to increase the assembly cost, therefore it is argued that a more low cost coarse assembly process must be developed to achieve an overall cost-effective assembly alternative compared to the conventional assembly methods. In general, assembly cost is lower if the required accuracy to be reached is also lower. Unfortunately, only in certain degrees of freedom this accuracy demand is lowered by the application of product-internal assembly functions. This does not permit the use of cheaper, less accurate assembly methods for the overall coarse assembly process. A possible solution is the use of features in the product that assist in achieving the required positioning accuracies, thus by obtaining the accuracy from the fabrication process instead of the assembly process. This approach does enable a further decrease of the assembly cost. Since MEMS-based technology is employed for realising the product-internal assembly functions, it may not involve much additional cost and effort on the fabrication side to include also such features to assist in achieving the desired accuracies in the coarse assembly process.

For the accurate fabrication of passive alignment structures, two technologies are available, both based on lithography for reaching the necessary (in-plane) dimensional accuracy:

- Alignment with the use of mechanical stops (compliant positioning)

- Alignment with the use of liquid surface tension (fluid self-alignment)

A mechanical stop can be defined as an embedded functional surface in a part to be assembled which constrains the fine motion between the part to be assembled and the sub-assembly during alignment. For each degree of freedom two contact areas and one alignment motion need to be defined. The contact areas have to be mated in the fine motion step by compliant actuation forces.

Alignment accuracy can also be increased using surface tension. In order to join two parts together, bonding materials like solder or adhesives need to be in a liquid phase. Surface tension forces occur, which can cause relative movement between parts to be assembled. By smart positioning of wettable surfaces during the fabrication process and by accurate control on the volume of the bonding material, surface tension within the liquid can be used for accurate positioning of small parts during the coarse assembly process (see Figure 6). Earlier-performed research ${ }^{4-10}$ has shown that surface tension forces within solder are able to align parts with high accuracies. Parts with an initial alignment of $\pm 20 \mu \mathrm{m}$ can be aligned up to $\pm 1 \mu \mathrm{m}$ and $0.1^{\circ}$ 
accuracies in the in plane $(\mathrm{x}, \mathrm{z})$ directions and $\pm 0.5 \mu \mathrm{m}$ in $\mathrm{y}$-direction (height).
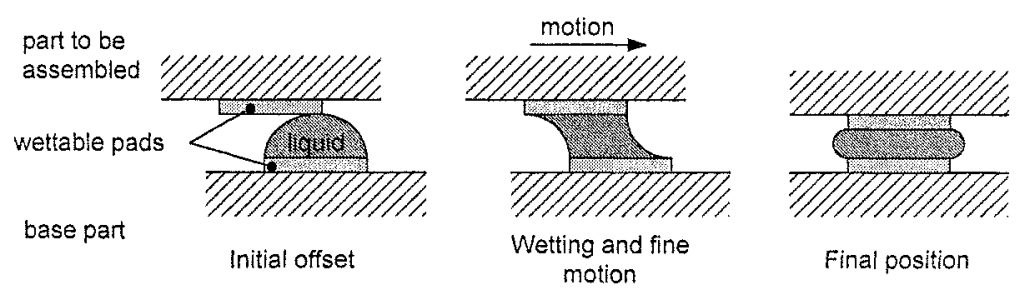

Figure 6. Alignment using liquid surface tension.

In various researches the above two technologies have been applied in silicon to attain high alignment accuracies for optical applications. The silicon base parts in these researches are generally referred to as silicon optical bench (SiOB) or silicon waferboard..$^{6,8,11-14}$ The present research could be considered as an extension to this concept, in which also active assembly functionality is integrated with the product. In the specific case considered as well both mechanical stops and fluid self-alignment have been utilised.

\subsubsection{Part relation tolerances}

Three main part relations need to be established:

- Assembly of the actuator part to the base part;

- Laser diode to base part assembly;

- Fibre insertion into the actuator part.

Assembly of the actuator and of the laser diode are proposed to be performed using solder self-alignment techniques. Wettable solder pads are to be integrated into the actuator part and the laser diode as well as in the silicon base part. Integrating fluid self-alignment features using MEMS technology is simpler than creating mechanical stops with comparable outof-plane accuracy. This is the reason that for the two planar part relations (laser diode to base part and actuator part-base part) this option has been selected. For the fibre-actuator part relation mechanical stops are more appropriate due to the more complex contact interface and the fact that it is very difficult to create high accuracy alignment features in the fibre part. Due to the specific characteristics of the fibre part it is only possible to constrain the position in four degrees of freedom $\left(x, y, \theta_{x}\right.$ and $\left.\theta_{y}\right)$. Tolerance build-up in these directions between chip and fibre are expected to be around $-0.5 \mu \mathrm{m}$ to $0 \mu \mathrm{m}$ and $-0.1^{\circ}$ to $0^{\circ}$ respectively due to a deliberate alignment 
of the fibre relative to the actuator end effector with a negative offset (so that the parts in all cases make contact with each other).

In the specific case under consideration a lensed fibre tip is used. This tip can either be rotation symmetric or non-rotation symmetric. Build-up of tolerance in the $\theta_{z}$-direction can be neglected for rotation symmetric fibre ends; for non-rotation symmetric fibre ends the angular misalignment in $\theta_{2}$ is not allowed to exceed $\pm 10^{\circ}$, which is not considered critical. Tolerances on the fabrication of the fibre tip generally are so large that mechanical stops cannot be used for successfully achieving the desired positioning accuracy in the longitudinal $(z)$ direction. Instead, for achieving the critical alignment tolerance in $z$-direction of $\pm 0.5 \mu \mathrm{m}$ of the fibre tip relative to the laser diode facet (optimal offset $4-5 \mu \mathrm{m}$ ) still a highly accurate insertion is needed, which is an undesired situation. The fibre could be maintained in place for example by mechanical clamps integrated in the actuator chip. After this final coarse assembly step the alignment of the fibre tip in $\mathrm{x}$ and $\mathrm{y}$-direction can be performed actively using the product-internal assembly functions.

An overview of the attainable coarse assembly tolerance build-up for the three main part relations is presented in Table 2. A schematic side view of the individual parts to be assembled is given in Figure 7 . The attainable tolerances for the laser diode and the actuator part relative to the silicon base part depend entirely on the fluid self-alignment process and are taken from literature.

Table 2. Overview of attainable tolerances coarse assembly process

\begin{tabular}{|c|c|c|c|c|c|}
\hline DOF & $\begin{array}{l}\sum \mathbf{i}_{\text {CA, allow }} \\
\text { Maximal allowed } \\
\text { tolerance build- } \\
\text { up of coarse } \\
\text { assenbly process }\end{array}$ & $\begin{array}{l}\quad \mathbf{i}_{\text {CA }} \\
\text { Actuator - } \\
\text { base part } \\
\text { (solder self- } \\
\text { alignment) }\end{array}$ & $\begin{array}{c}\mathbf{i}_{\mathrm{CA}_{A}} \\
\text { Laser diode } \\
\text { - base part } \\
\text { (solder } \\
\text { self- } \\
\text { alignment) }\end{array}$ & $\begin{array}{c}\mathbf{i}_{\mathrm{CA}} \\
\text { Fibre - } \\
\text { actuator } \\
\text { (enforced } \\
\text { by actuator } \\
\text { design) }\end{array}$ & $\begin{array}{c}\Sigma \mathbf{I}_{\text {CA, attain }} \\
\text { Attainable } \\
\text { tolerance build-up } \\
\text { coarse assembly } \\
\text { process }\end{array}$ \\
\hline $\mathrm{x}$ & $\pm 5.5 \mu \mathrm{m}$ & $\pm 1 \mu \mathrm{m}$ & $\pm 1 \mu \mathrm{m}$ & $\begin{array}{c}-0.5 \mu \mathrm{m} \\
0\end{array}$ & $\begin{array}{l}-2.5^{\circ} \\
+2^{\circ}\end{array}$ \\
\hline$y$ & $\pm 2.5 \mu \mathrm{m}$ & $\pm 0.5 \mu \mathrm{m}$ & $\pm 0.5 \mu \mathrm{m}$ & $\begin{array}{c}-0.5 \mu \mathrm{m} \\
0\end{array}$ & $\begin{array}{l}-1.5^{\circ} \\
+1^{\circ}\end{array}$ \\
\hline $\mathrm{z}$ & $\pm 0.5 \mu \mathrm{m}$ & $\pm 1 \mu \mathrm{m}$ & $\pm 1 \mu \mathrm{m}$ & & $\pm 2 \mu \mathrm{m}$ \\
\hline$\theta_{\mathrm{x}}$ & $-1^{\circ}+1.5^{\circ}$ & Insignificant & $\pm 0.1^{\circ}$ & $\begin{array}{c}-0.1^{\circ} \\
0\end{array}$ & $\begin{array}{l}-0.2^{\circ} \\
+0.1^{\circ}\end{array}$ \\
\hline$\theta_{\mathrm{y}}$ & $-1^{\circ}+1.5^{\circ}$ & Insignificant & $\pm 0.3^{\circ}$ & $\begin{array}{c}-0.1^{\circ} \\
0\end{array}$ & $\begin{array}{l}-0.4^{\circ} \\
+0.3^{\circ}\end{array}$ \\
\hline$\theta_{\mathrm{z}}$ & $\pm 10^{\circ}$ & Insignificant & $\pm 0.1^{\circ}$ & & $\pm 0.1^{\circ}$ \\
\hline
\end{tabular}



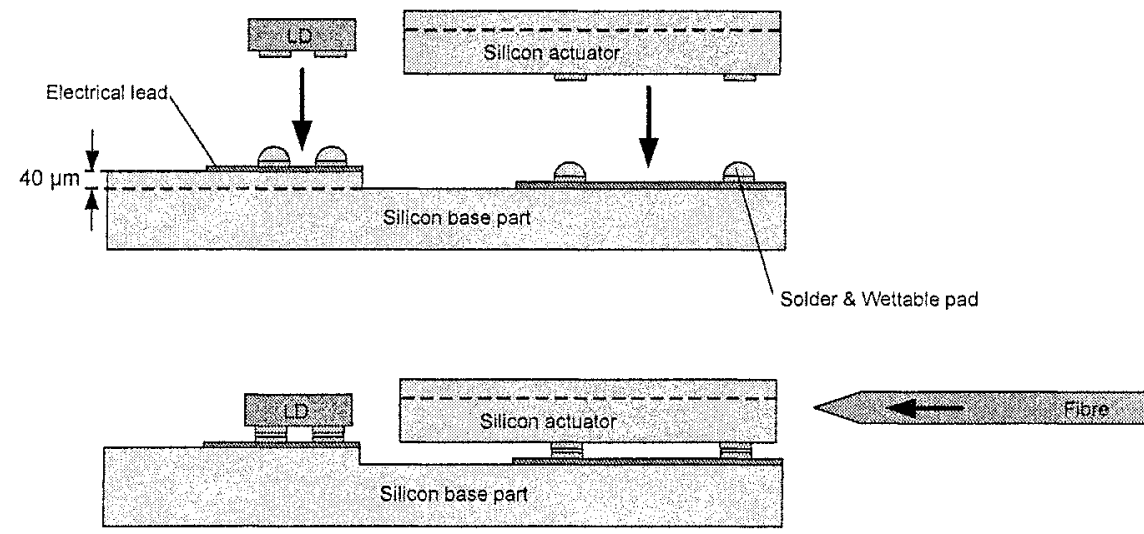

Figure 7. Schematic order coarse assembly steps.

\section{CONCLUSIONS}

For specific applications the method of micro-assembly by means of product-internal assembly functions could be a promising alternative to conventionally used methods for micro-assembly tasks. A significant degree of design complexity is involved, especially in realising the desired positioning accuracies, due to the existence of a high level of interrelations between a number of closely connected issues that play a role within this assembly method.

Two basic approaches can be distinguished for obtaining the desired positioning accuracies during the coarse assembly process: (1) obtaining the desired coarse positioning accuracy from the assembly process, or (2) from the fabrication process. The last is considered essential for achieving high positioning accuracies at relatively low cost.

At this stage no optimal overall assembly solution has been found for this specific case under investigation. In this research a sequential approach has been taken from fine to coarse assembly; a more optimal result should be feasible in a concurrent development approach.

\section{ACKNOWLEDGEMENT}

This research belongs to the Delft Centre for Mechatronics and Microsystems of the TU Delft. It is funded by the Dutch government programme IOP Precision Engineering as part of the project IPT02310 Technologies for in-package optical fibre-chip coupling. 


\section{REFERENCES}

1. M. Tichem and B. Karpuschewski, Structuring of micro-assembly methods, CD-ROM Proceedings of the $33^{\text {rd }}$ Int. Symp. on Robotics, Stockholm (October 7-11, 2002).

2. R.R.A. Syms, H. Zou, and J. Stagg, Robust latching MEMS translation stages for microoptical systems, J. Micromech. Microeng. 14, 667-674 (2004).

3. V.A. Henneken, S.P.W. van den Bedem, M. Tichem, B. Karpuschewski, and P.M. Sarro, Design of in-package MST-based actuators for micro-assembly, CD-ROM Proceedings $7^{\text {th }}$ SAFE workshop, Veldhoven, the Netherlands (November 25-26, 2004).

4. Q. Tan, Y.C. Lee, Soldering technology for optoelectronic packaging, Proceedings IEEE ECTC, pp. 26-36 (1996).

5. A.R. Mickelson, N.R. Basavanhally, Y.C. Lee, Optoelectronic Packaging (John Wiley \& Sons, New York, 1997).

6. R.M. Edge, Flip-chip solder bond mounting of laser diodes, Electron. Lett. 27(6), 499-501 (1991).

7. J. Sasaki, M. Itoh, T. Tamanuki, et al., Multiple-chip precise self-aligned assembly for hybrid integrated optical modules using Au-Sn solder bumps, IEEE Trans. Adv. Pack. 24(4), 569-575 (2001).

8. M. Itoh, Passive alignment on Si optical bench using Au-Sn solder bumps, Proceedings IEEE LEOS $10^{\text {th }}$ Annual Meeting, pp. 126-127 (1997).

9. W. Lin, S.K. Patra, Y.C. Lee, Design of solder joints for self-aligned optoelectronic assemblies, IEEE Trans. Comp. Pack. Manuf. B 18(3), 543-551 (1995).

10.W. Pittroff, J. Barnikow, A. Klein, P. Kurpas, et al., Flip chip mounting of laser diodes with $\mathrm{Au} / \mathrm{Sn}$ solder bumps: bumping, self-alignment and laser behavior, Proceedings IEEE ECTC, pp. 1235-1241 (1997).

11.C.A. Armiento, A.J. Negri, M.J. Tabaski, R.A. Boudreau, et al., Gigabit transmitter array modules on silicon waferboard, IEEE Trans. Comp. Hybr. Manuf. 15(6), 1072-1080 (1992).

12.R.M. Bostock, J.D. Collier, R-J.E. Jansen, R. Jones, et al., Silicon nitride microclips for the kinematic location of optic fibres in silicon V-shaped grooves, J. Micromech. Microeng. 8, 343-360 (1998).

13.C. Strandman, Y. Backlund, Bulk silicon holding structures for mounting of optical fibers in V-grooves, J. Microelectromech. Syst. 6(1), 35-40 (1997).

14.C. Strandman, Y. Backlund, Passive and fixed alignment of devices using flexible silicon elements formed by selective etching, $J$. Micromech. Microeng. 8, 39-44 (1998). 